\title{
Estudo da frequência dos alelos de HLA-DRB1 em pacientes brasileiros com artrite reumatoide
}

\author{
Magali Justina Gómez Usnayo', Luis Eduardo Coelho Andrade², \\ Renata Triguenho Alarcon 3 , Juliana Cardoso Oliveira ${ }^{4}$, Gustavo Milson Fabrício Silva ${ }^{4}$, \\ Izidro Bendet ${ }^{5}$, Rufus Burlingame ${ }^{6}$, Luis Cristóvão Porto ${ }^{7}$, Geraldo da Rocha Castelar Pinheiro ${ }^{8}$
}

\begin{abstract}
RESUMO
Os alelos HLA-DRB1, que codificam uma sequência de aminoácidos (QKRAA/QRRAA/RRRAA) nas posições 70 a 74 da terceira região hipervariável da cadeia $\beta 1$ do gene DRB1, denominada epítopo compartilhado (EC), estão associados a maior suscetibilidade e gravidade para artrite reumatoide (AR) em diversas populações. Objetivo: Determinar a frequência dos alelos HLA-DRB1 em pacientes brasileiros com AR, e sua associação a fator reumatoide (FR) e anticorpos antipeptídeos citrulinados (ACPA). Material e métodos: Foram incluídos 412 pacientes com AR e 215 controles. A tipificação HLA-DRB1 foi realizada pela reação em cadeia da polimerase (PCR) usando primers específicos e hibridização com oligonucleotídeos de sequência específica (SSOP). A pesquisa de ACPA foi determinada pela técnica de ELISA, e a do FR por nefelometria. Para análises estatísticas foram utilizados os testes do qui-quadrado e $t$ de Student e a regressão logística. Resultados: Alelos HLA-DRB1*04:01, *04:04 e *04:05 associaram-se à AR $(\mathrm{P}<0,05)$ ); a despeito do amplo intervalo de confiança, vale a pena ressaltar a associação observada entre o alelo DRB1*09:01 e a doença $(\mathrm{P}<0,05)$. Alelos HLA-DRB1 EC+ foram observados em 62,8\% dos pacientes e em 31,1\% do grupo-controle (OR 3,62; P $<0,001)$ e estiveram associados a ACPA (OR 2,03; P < 0,001). Alelos DRB1-DERAA mostraram efeito protetor para AR (OR 0,42; $\mathrm{P}<0,001)$. Conclusão: Em uma amostra de pacientes brasileiros com AR de etnia majoritariamente mestiça, alelos HLA-DRB1 EC+ estiveram associados à suscetibilidade à doença e à presença de ACPA.
\end{abstract}

Palavras-chave: HLA-DRB1, epítopo compartilhado, artrite reumatoide, polimorfismo gênico, imunogenética.

(C) 2011 Elsevier Editora Ltda. Todos os direitos reservados.

\section{INTRODUÇÃO}

A artrite reumatoide (AR) é uma doença sistêmica crônica, caracterizada pelo acometimento inflamatório da membrana sinovial das articulações, levando à destruição óssea e cartilaginosa. Sua prevalência na população adulta mundial é de $0,5 \%$ a $1 \%$, e no Brasil é de $0,46 \% .{ }^{1}$ Apresenta pico de incidência entre a quarta e a sexta décadas, e é duas a três vezes mais frequente em mulheres que em homens. ${ }^{2}$

Embora sua etiologia permaneça desconhecida, ${ }^{3}$ vários estudos sugerem que uma combinação de fatores genéticos e ambientais esteja envolvida. $\mathrm{O}$ fator genético contribui com cerca de $60 \%$ da suscetibilidade para AR. ${ }^{4}$ Embora o papel da hereditariedade não esteja completamente compreendido,

Recebido em 19/6/2011. Aceito, após revisão, em 21/6/2011. Os autores declaram a inexistência de conflitos de interesse. Comitê de Ética: CEP-HUPE 2169. Apoio financeiro: Centro de Estudos em Reumatologia Pedro Ernesto - CERPE.

Disciplina de Reumatologia do Hospital Universitário Pedro Ernesto - UERJ.

1. Mestranda do Programa de Pós-graduação em Ciências Médicas da Universidade Estadual do Rio de Janeiro - UERJ

2. Professor-Associado da Disciplina de Reumatologia da Universidade Federal de São Paulo - UNIFESP

3. Doutoranda do Programa de Pós-graduação em Reumatologia da UNIFESP

4. Biólogo-Assistente do Laboratório de Histocompatibilidade e Criopreservação da UERJ e Doutorando do Programa de Pós-graduação em Biologia Humana e Experimental da UERJ

5. Consultor Científico de Imunologia do Sérgio Franco Medicina Diagnóstica - DASA

6. Cientista sênior da INOVA Diagnostics, Inc. San Diego, California, EUA

7. Professor Titular do Departamento de Histologia e Embriologia e Coordenador do Laboratório de Histocompatibilidade e Criopreservação da UERJ

8. Professor Adjunto da Disciplina de Reumatologia da Faculdade de Ciências Médicas da UERJ

Correspondência para: Geraldo da Rocha Castelar Pinheiro. Boulevard 28 de Setembro, 77 - sala 333 -Vila Isabel. CEP: $20551-030$. Rio de Janeiro, RJ, Brasil.

Telefone: +55 21 2868-8216. Email: geraldo.castelar@gmail.com 
o fator de risco predominante, responsável por $30 \%$ a $50 \%$ do componente genético, parece estar ligado aos antígenos leucocitários humanos (HLA, do inglês, human leukocyte antigen). O HLA localiza-se no complexo principal de histocompatibilidade (MHC, do inglês, major histocompatibility complex) presente no braço curto do cromossoma 6 (6p21.3). ${ }^{5}$ Um grupo de alelos do lócus HLA-DRB1 (DRB1*01:01, DRB1*01:02, DRB1*04:01, DRB1*04:04, DRB1*04:05, DRB1*04:08, DRB1*04:10, DRB1*10:01, DRB1*14:02) codifica uma sequência de aminoácidos compartilhada (QKRAA/QRRAA/RRRAA, onde $\mathrm{Q}=$ glutamina; $\mathrm{K}$ = lisina; $\mathrm{R}=$ arginina e $\mathrm{A}=$ alanina), localizada no sulco de ligação ao peptídeo, nas posições 70 a 74 da terceira região hipervariável da molécula HLA-DR, sequência esta denominada de epítopo compartilhado (EC). ${ }^{6}$

Acredita-se que o EC esteja envolvido na patogênese da AR, por servir de local de ligação no processo de apresentação de peptídeos artritogênicos para as células T $\mathrm{CD} 4^{+}$envolvidas na resposta imunoinflamatória dessa doença. Além disso, o EC pode estar envolvido no processo de indução de algumas células $\mathrm{B}$ a diferenciarem-se em plasmócitos, levando à formação dos anticorpos antipeptídeos citrulinados (ACPA, do inglês, anti-citrullinated peptides antibody). ${ }^{7}$ Além do seu papel na suscetibilidade para $\mathrm{AR}$, alelos que contêm as sequências do EC (principalmente homozigotos) estão associados às formas mais graves da enfermidade e às manifestações extra-articulares, ${ }^{8}$ bem como à presença de doença erosiva. ${ }^{9}, 10$

Estudos realizados em diversos grupos étnicos mostraram a existência de variações consideráveis com relação à associação dos alelos de HLA-DRB1 com a suscetibilidade à AR. ${ }^{11}$ Alelos HLA-DRB1*04:01 e *04:04 estão associados à suscetibilidade à doença em indivíduos caucasianos do norte da Europa e dos Estados Unidos; ${ }^{12}$ o DRB $1 * 04: 05$ em coreanos, japoneses e chineses; ${ }^{13,14}$ o DRB $1 * 01: 01$ e o *10:01 em gregos, espanhóis e judeus israelenses; ${ }^{15,16}$ o DRB1*14:02 em índios nativos americanos, peruanos ${ }^{17,18}$ e equatorianos; ${ }^{19}$ o DRB1*04:04 em colombianos e argentinos. ${ }^{20,21}$ No Brasil, Bertolo et al. ${ }^{22}$ observaram associação da AR ao DRB1*01:01 e *1:02 em 65 pacientes caucasianos. Mais recentemente, Louzada-Junior et al. ${ }^{23}$ encontraram associação da doença aos alelos DRB1*04:01, *04:04, *04:05, *01:01 e *10:01 em 140 pacientes, de maioria étnica caucasiana.

Em contrapartida, os alelos HLA-DRB1, com uma sequência de aminoácidos comum, DERAA ( $\mathrm{D}$ = ácido aspártico, $\mathrm{E}=$ ácido glutâmico, $\mathrm{R}=$ arginina, $\mathrm{A}=$ alanina), expressa nos alelos DRB1*01:03, *04:02, *11:02, *11:03, *13:01, 13:02 e *13:04, parecem estar associados a menor risco para o desenvolvimento da $\mathrm{AR}$, independentemente da presença do EC.
A presença desses alelos parece também ser protetora contra doença erosiva grave, mesmo em pacientes com ACPA. ${ }^{24,25}$

Os principais marcadores imunológicos da $A R$, fator reumatoide (FR) e ACPA, aparentemente também estão envolvidos na patogênese da sinovite reumatoide. A presença de títulos elevados de ambos foi associada à doença mais agressiva e erosiva. ${ }^{26,27}$

Alguns estudos em pacientes com AR têm mostrado resultados contraditórios com relação à associação do EC e à positividade do FR. ${ }^{28,29}$ Ao mesmo tempo, parece haver uma associação dos alelos EC+ apenas em pacientes com AR que apresentam ACPA positivos, ${ }^{30} \mathrm{e}$ que essa associação seria mais intensa com ACPA que com a própria AR. ${ }^{31}$ Tal associação sugere que os alelos EC poderiam influenciar a apresentação do antígeno levando à produção de ACPA. Mais recentemente, foi observado que o risco conferido pelo tabagismo, principal fator ambiental, é particularmente elevado nos indivíduos que possuem alelos HLA-DR EC+ com ACPA. ${ }^{32}$

Tendo em conta a diversidade dos resultados da literatura referentes à associação de alelos HLA-DRB1 à AR em diferentes etnias, o objetivo do presente estudo foi determinar a associação entre esses alelos e a AR, incluindo a presença de FR e/ou ACPA em uma população de pacientes brasileiros altamente miscigenados.

\section{MATERIAL E MÉTODOS}

\section{Pacientes e controle}

Nosso estudo foi tipo caso-controle, em que foram incluídos pacientes com AR. Todos preencheram pelo menos quatro dos sete critérios para classificação diagnóstica da AR estabelecidos pelo American College of Rheumatology (ACR). ${ }^{33}$ Os pacientes selecionados não apresentavam outras doenças autoimunes e eram acompanhados regularmente nos ambulatórios de AR das disciplinas de Reumatologia do Hospital Universitário Pedro Ernesto, da Universidade Estadual do Rio de Janeiro (UERJ), e do Hospital São Paulo, da Universidade Federal de São Paulo (UNIFESP), de outubro de 2007 a agosto de 2009. No grupo-controle foram incluídos indivíduos doadores voluntários de medula óssea, de campanhas de doação realizadas em diferentes bairros do Rio de Janeiro, de maio de 2008 a novembro de 2009. A faixa etária dos doadores foi de 18 a 55 anos, eram de ambos os gêneros e não apresentavam queixa atual ou passada de artrite. Também não foram incluídas pessoas com história familiar de $\mathrm{AR}$ ou outras doenças autoimunes em parentes de primeiro grau. Foram selecionados os maiores de 30 anos, para minimizar o viés de análise quanto ao possível diagnóstico futuro dessa doença neste grupo. 
Após serem informados sobre a natureza do estudo e terem assinado o Termo de Consentimento Livre e Esclarecido, os participantes foram submetidos a uma entrevista clínica, com ficha padronizada para os dois grupos. Foram registrados dados demográficos para ambos os grupos, além de características clínicas para o grupo dos pacientes. Ambos os grupos tiveram amostra de sangue coletada para tipificação do HLA. O grupo de pacientes foi submetido, também, à detecção de autoanticorpos (FR e ACPA).

Os grupos foram pareados para gênero e etnia. A atribuição da origem étnica foi realizada pelo mesmo investigador, após solicitar aos pacientes e doadores voluntários dados sobre sua ascendência. Foi considerado de etnia negra ou caucasiana aquele indivíduo com os quatro avôs com ascendência negra ou caucasiana, respectivamente, e mestiço quando pelo menos um dos avôs era de etnia diferente. Não foram incluídos descendentes de asiáticos, com a finalidade de evitar viés de seleção.

Em ambos os grupos foram excluídos os indivíduos que não possuíam quantidade de DNA ou soro suficientes para uma adequada análise da tipificação do HLA ou dosagem dos autoanticorpos. Não foram incluídas também pessoas cuja tipificação mostrou resultado ambíguo.

\section{Tipificação HLA-DRB1}

Os alelos HLA-DRB1 foram determinados para todos os pacientes com AR e controles. A tipificação HLA foi realizada pela reação em cadeia da polimerase (PCR) usando primers específicos e hibridização com oligonucleotídeos de sequência específica ((SSOP, do inglês, sequence-specific oligonucleotide probe) (One Lambda Inc., Canoga Park, CA, EUA).

\section{Definição dos alelos HLA-DRB1 de risco para AR}

Todo indivíduo com tipificação para os alelos HLA-DRB1*01:01, *01:02, *04:01, *04:04, *04:05, *04:08, *04:10, *10:01 e *14:02 foi considerado portador do epítopo compartilhado $(\mathrm{EC}+)$, podendo ser em uma dose $(\mathrm{EC}+/-)$ ou dose dupla $(\mathrm{EC}+/+)$. Associações de risco para AR entre alelos HLADRB1 foram realizadas a partir de combinações genotípicas estabelecidas na ausência de EC (-/-), na presença de uma dose do EC (+/-) ou de dose dupla do EC (+/+). A associação do HLA-DRB1 com as características sorológicas nos pacientes com AR também foi realizada.

\section{Definição dos alelos HLA-DRB1 de proteção para AR}

Todo indivíduo com tipificação para os alelos HLA-DRB1*01:03, $* 04: 02, * 11: 02, * 11: 03, * 13: 01, * 13: 02$ e *13:04 foi definido como portador da sequência DERAA (protetora), podendo ser em uma dose ou em dose dupla.

Os indivíduos cuja tipificação compreendia alelos não pertencentes à sequência do EC ou à sequência DERAA foram definidos com $\mathrm{X}$, podendo estar em uma dose ou em dose dupla.

Assim, formaram-se seis grupos de indivíduos para análise, de acordo com a presença dos alelos DRB1:

Grupo A: dose dupla (homozigotos) para EC (EC/EC);

Grupo B: dose única (heterozigoto) alelo EC (EC/X);

Grupo C: dose única de EC e de DERAA (EC/DERAA);

Grupo D: ausência de EC e de DERAA (X/X);

Grupo E: dose única de DERAA (DERAA/X);

Grupo F: dose dupla de DERAA (DERAA/DERAA).

\section{Determinação de FR e ACPA}

A detecção do ACPA foi realizada empregando o QUANTA Lite $^{\circledR}$ de $2^{\text {a }}$ geração CCP ELISA (INOVA Diagnostics, Inc - San Diego, EUA) de acordo com as instruções do fabricante. A quantificação de FR IgM foi realizada pela técnica de nefelometria (Dade Behring Marburg GmbH Alemanha). Para o FR IgM foram considerados positivos títulos $>20 \mathrm{UI} / \mathrm{mL}$, e elevados quando $>100 \mathrm{UI} / \mathrm{mL}$. Para o ACPA foram considerados positivos títulos $>20 \mathrm{U} / \mathrm{mL}$, e elevados quando $>60 \mathrm{U} / \mathrm{mL}$.

\section{Análise estatística}

Os dados foram analisados utilizando o programa Epi Info 6. A análise da associação entre as variáveis categóricas foi feita pelo teste do qui-quadrado (com correção de Yates) ou teste exato de Fisher. As variáveis quantitativas com distribuição normal foram testadas utilizando-se teste $t$ de Student para amostras independentes ou Mann-Whitney e análises de variância. Valores de $\mathrm{P}<0,05$ foram considerados estatisticamente significativos. Análise de regressão logística foi utilizada para categorizar o risco de associação dos alelos HLA-DRB1.

\section{RESULTADOS}

Foram incluídos no estudo 412 (95\%) dos 430 pacientes com AR e 215 (74\%) dos 290 controles doadores voluntários de medula óssea. As características de gênero e etnia foram similares em ambos os grupos $(\mathrm{P}=0,722$ e $\mathrm{P}=0,552)$. Os demais dados demográficos, clínicos e laboratoriais dos pacientes com AR e do grupo-controle encontram-se descritos na Tabela 1. 


\section{Associação de grupos alélicos HLA-DRB1 com AR}

A Tabela 2 mostra a distribuição das frequências de grupos alélicos do HLA-DRB1 e a associação à suscetibilidade e proteção para AR entre 412 pacientes com AR e 215 voluntários do grupo-controle. Alelos HLA-DRB1*04 $(\mathrm{OR}=2,69)$,
*09 $(\mathrm{OR}=5,19) \mathrm{e} * 14(\mathrm{OR}=2,26)$ estiveram associados à suscetibilidade à $\mathrm{AR}(\mathrm{P}<0,05)$, enquanto os alelos DRB $1 * 11$ $(\mathrm{OR}=0,45) \mathrm{e} * 13(\mathrm{OR}=0,53)$ estiveram associados à proteção ao desenvolvimento da AR $(\mathrm{P}<0,05)$. De forma semelhante, quando realizamos a tipificação dos alelos HLA-DRB1 por alta

\section{Tabela 1}

Caracterização demográfica e clínico-laboratorial de pacientes com AR e grupo-controle

\begin{tabular}{llll}
\hline Variável & AR $(\mathbf{n}=\mathbf{4 1 2})$ & Controle $(\mathbf{n}=\mathbf{2 1 5})$ & P \\
\hline Idade (média \pm DP, anos) & $51,8 \pm 11,5 / 52,5$ & $42,6 \pm 5,4$ & $<0,001$ \\
Gênero feminino (\%) & $376(90,8)$ & $198(92,1)$ & 0,722 \\
\hline Etnia & & & 0,552 \\
$\quad$ Mestiços (\%) & $272(66)$ & $147(68,4)$ & $58(27)$ \\
$\quad$ Brancos (\%) & $111(27)$ & $10(4,7)$ & - \\
Negros (\%) & $29(7)$ & - & - \\
Tempo de doença (média \pm DP, anos) & $9,2 \pm 7,5$ & - \\
FR+ (\%) & $257(62,5)$ & - \\
Titulação do FR (média \pm DP, Ul/mL) & $288 \pm 668$ & \\
ACPA+ (\%) & $294(71,3)$ & $135 \pm 51,5$ & \\
TTitulação do ACPA (média \pm DP, U/mL) &
\end{tabular}

AR: artrite reumatoide; ACPA: anticorpo antipeptídeo citrulinado; FR: fator reumatoide; DP: desvio-padrão.

Tabela 2

Frequências dos grupos alélicos do HLA-DRB1 (baixa resolução) em pacientes com AR e grupo-controle

\begin{tabular}{|c|c|c|c|c|c|}
\hline Grupo alélico & $A R=412, n(\%)$ & Controle $=215, n(\%)$ & OR & $95 \%$ IC & $\mathbf{P}$ \\
\hline$* 01$ & $113(14)$ & $45(10,5)$ & 1,36 & $0,93-2,00$ & NS \\
\hline *03 & $73(9)$ & $54(12,5)$ & 0,68 & $0,46-1,00$ & NS \\
\hline$* 04$ & $186(22,5)$ & $42(10)$ & 2,69 & $1,86-3,92$ & 0,001 \\
\hline *07 & $79(9,5)$ & $52(12)$ & 0,77 & $0,52-1,14$ & NS \\
\hline$* 08$ & $38(5)$ & $20(5)$ & 0,99 & $0,55-1,79$ & NS \\
\hline *09 & $29(3,5)$ & $3(1)$ & 5,19 & $1,50-21,49$ & 0,004 \\
\hline$* 10$ & $27(3)$ & $14(3)$ & 1,01 & $0,50-2,04$ & NS \\
\hline$* 11$ & $53(6)$ & $57(13)$ & 0,45 & $0,30-0,68$ & 0,001 \\
\hline$* 12$ & $12(1,5)$ & $9(2)$ & 0,69 & $0,27-1,79$ & NS \\
\hline *13 & $85(10)$ & $77(18)$ & 0,53 & $0,37-0,75$ & 0,001 \\
\hline *14 & $38(5)$ & $9(1)$ & 2,26 & $1,04-5,09$ & 0,038 \\
\hline *15 & $64(8)$ & $43(10)$ & 0,76 & $0,50-1,16$ & NS \\
\hline *16 & $27(3)$ & $11(2,5)$ & 1,29 & $0,61-2,80$ & NS \\
\hline TOTAL & 824 & 430 & & & \\
\hline
\end{tabular}

AR: artrite reumatoide; OR: razão de risco; NS: não significante. 
Tabela 3

Frequência alélica do HLA-DRB1 em pacientes com AR e grupo-controle

\begin{tabular}{|c|c|c|c|c|c|}
\hline Alelos HLA-DRB1 & $\mathrm{AR}=824, \mathrm{n}(\%)$ & Controle $=430, n(\%)$ & OR & $95 \%$ IC & $\mathbf{P}$ \\
\hline$* 01: 01$ & $68(8,3)$ & $26(6)$ & 1,40 & $0,86-2,29$ & NS \\
\hline *01:02 & $41(5)$ & $17(4)$ & 1,27 & $0,69-2,36$ & NS \\
\hline$* 01: 03$ & $4(0,5)$ & $2(0,5)$ & 1,04 & $0,16-8,22$ & NS \\
\hline *03 & $73(8,8)$ & $54(12,5)$ & 0,68 & $0,46-1,00$ & NS \\
\hline$* 04: 01$ & $41(5)$ & $8(1,9)$ & 2,76 & $1,23-6,44$ & 0,010 \\
\hline *04:02 & $14(1,7)$ & $5(1,2)$ & 1,47 & $0,49-4,70$ & NS \\
\hline$* 04: 03$ & $9(1,1)$ & $5(1,2)$ & 0,94 & $0,29-3,23$ & NS \\
\hline *04:04 & $42(5)$ & $6(1,4)$ & 3,80 & $1,53-10,0$ & 0,002 \\
\hline$* 04: 05$ & $48(5,8)$ & $10(2,3)$ & 2,60 & $1,25-5,53$ & 0,007 \\
\hline$* 04: 06$ & $3(0,4)$ & $1(0,2)$ & 1,57 & $0,15-39,2$ & NS \\
\hline$* 04: 07$ & $7(0,8)$ & $3(0,7)$ & 1,22 & $0,28-5,97$ & NS \\
\hline *04:08 & $12(1,5)$ & $0(0,0)$ & ND & ND & NS \\
\hline$* 04: 10$ & $3(0,4)$ & $0(0,0)$ & ND & ND & NS \\
\hline$* 04: 11$ & $7(0,8)$ & $4(0,9)$ & 0,91 & $0,24-3,72$ & NS \\
\hline$* 07$ & $79(9,6)$ & $52(12,1)$ & 0,77 & $0,52-1,14$ & NS \\
\hline$* 08$ & $38(4,6)$ & $20(4,7)$ & 0,99 & $0,55-1,79$ & NS \\
\hline *09:01 & $29(3,5)$ & $3(0,7)$ & 5,19 & $1,50-21,49$ & 0,004 \\
\hline *10:01 & $27(3,3)$ & $8(1,9)$ & 1,79 & $0,77-4,31$ & NS \\
\hline$* 11$ & $53(6,4)$ & $57(13,2)$ & 0,45 & $0,30-0,68$ & 0,001 \\
\hline *12 & $12(1,4)$ & $9(2,1)$ & 0,69 & $0,27-1,79$ & NS \\
\hline$* 13$ & $85(10,3)$ & $77(17,9)$ & 0,53 & $0,37-0,75$ & 0,001 \\
\hline *14:01 & $20(2,4)$ & $6(1,4)$ & 1,76 & $0,66-4,92$ & NS \\
\hline *14:02 & $18(2,2)$ & $3(0,7)$ & 3,18 & $0,88-13,63$ & NS \\
\hline *15 & $64(7,8)$ & $43(10)$ & 0,76 & $0,50-1,16$ & NS \\
\hline$* 16$ & $27(3,3)$ & $11(2,6)$ & 1,29 & $0,61-2,80$ & NS \\
\hline
\end{tabular}

AR: artrite reumatoide; OR: razão de risco; NS: não significante.

resolução (Tabela 3), a análise das frequências alélicas mostrou que os alelos *DRB1*04:01 (OR $=2,76),{ }^{*} 04: 04(\mathrm{OR}=3,80)$, $*$ 04:05 $(\mathrm{OR}=2,60) \mathrm{e} * 09: 01(\mathrm{OR}=5,19)$ estiveram associados à suscetibilidade à $\mathrm{AR}(\mathrm{P}<0,05)$.

\section{Associação dos alelos HLA-DRB1 do EC na suscetibilidade à $A R$}

A Tabela 4 mostra a distribuição de pacientes com AR e indivíduos do grupo-controle de acordo com a presença dos alelos HLA-DRB1 EC+. Observamos maior frequência de genótipos heterozigotos $(51 \%)$ que homozigotos (11\%) no grupo de pacientes, estando ambos associados à suscetibilidade à $\mathrm{AR}(\mathrm{OR}=2,90, \mathrm{P}<0,001$ e $\mathrm{OR}=2,27$, $\mathrm{P}<0,001$, respectivamente). Por fim, observamos frequência aumentada do EC no grupo de pacientes com AR $(62,8 \%)$ quando comparados com os controles $(31,1 \%)$, conferindo um $\mathrm{OR}=3,59(\mathrm{P}=0,05)$.

A análise de regressão logística dos dados dos pacientes com AR e grupo-controle com genótipos homozigotos e heterozigotos HLA-DRB1 com EC+ e DRB1 sem EC- mostrou risco aumentado para a doença de 3,86 vezes mais entre pacientes que apresentam genótipos homozigotos (IC 95\% 1,84-8,24; $\mathrm{P}<0,001$ ), e de 3,54 vezes mais entre os que apresentam genótipos heterozigotos (IC 95\% 2,40-5,21; $\mathrm{P}<0,001$ ). 


\section{Tabela 4}

Especificidades individuais de HLA-DRB1 associados à AR entre brasileiros de acordo com genótipos relacionados à presença do EC

\begin{tabular}{|c|c|c|c|c|c|}
\hline EC e genótipo & $A R=412, n(\%)$ & Controle $=215, n(\%)$ & OR & $95 \%$ IC & $\mathbf{P}$ \\
\hline Alelos EC+/EC- & $210(51)$ & $56(26)$ & 2,90 & $1,99-4,23$ & $<0,001$ \\
\hline *01:01 & $48(11,6)$ & $18(8,3)$ & 1,44 & $0,79-2,65$ & 0,257 \\
\hline *04:01 & $23(5,5)$ & $6(2,7)$ & 2,06 & $0,78-5,74$ & 0,167 \\
\hline *04:04 & $27(6,5)$ & $4(1,8)$ & 3,70 & $1,21-12,54$ & 0,017 \\
\hline *04:08 & $8(1,9)$ & - & NS & - & 0,092 \\
\hline *04:10 & $3(0,7)$ & - & NS & - & 0,519 \\
\hline *10:01 & $21(5)$ & $7(3,2)$ & 1,60 & $0,63-4,20$ & 0,392 \\
\hline *14:02 & $13(3,1)$ & $3(1,3)$ & 2,30 & $0,61-10,28$ & 0,289 \\
\hline Alelos EC+/ EC+ & $45(11)$ & $11(5)$ & 2,27 & $1,11-4,77$ & 0,023 \\
\hline$* 04: 04 / * 04: 04$ & $3(0,7)$ & - & NS & NS & NS \\
\hline$* 04: 05 / * 04: 05$ & $1(0,2)$ & - & NS & NS & NS \\
\hline *14:02/*14:02 & $1(0,2)$ & - & NS & NS & NS \\
\hline *01:01/*04:01 & $8(1,9)$ & $2(0,9)$ & 2,11 & $0,41-14,50$ & 0,532 \\
\hline$* 01: 01 / * 04: 04$ & $3(0,7)$ & - & NS & NS & NS \\
\hline *01:01/*04:05 & $3(0,7)$ & $2(0,9)$ & NS & NS & NS \\
\hline *01:01/*10:01 & $2(0,5)$ & - & NS & NS & NS \\
\hline *01:02/*04:01 & $2(0,5)$ & - & NS & NS & NS \\
\hline
\end{tabular}

AR: artrite reumatoide; EC+: epítopo compartilhado positivo; EC-: epítopo compartilhado negativo; OR: razão de risco; NS: não significante.

\section{Associação dos alelos HLA-DRB1-DERAA na proteção ao desenvolvimento da AR}

Avaliando a influência da presença de alelos DERAA, observamos que 79 (19\%) pacientes com AR e 78 (36\%) controles possuem os alelos de HLA-DRB1 codificando DERAA, o que indica que sua presença confere proteção para $\mathrm{AR}(\mathrm{OR}=0,42$; IC 95\% 0,28-0,61; $\mathrm{P}<0,001)$.
O efeito de alelos DERAA na ausência de alelos EC foi avaliado comparando os grupos D (X/X) e E (X/DERAA) mais o grupo $\mathrm{F}$ (DERAA/DERAA). Indivíduos com DERAA positivo tiveram menor risco para desenvolver $\mathrm{AR}(\mathrm{OR}=0,56$; IC 95\% 0,34-0,92; $\mathrm{P}=0,021$ ).

A comparação entre os grupos B (EC/X) e C (EC/DERAA) revelou que, na presença de um alelo EC, a codificação de alelos DERAA conferiu menor suscetibilidade para AR, embora sem significância estatística $(\mathrm{OR}=0,60$; IC 95\% 0,28-1,29; $\mathrm{P}=0$,216) (Tabela 5). 


\section{Tabela 5}

Frequências de genótipos HLA-DRB1 de suscetibilidade (EC) e proteção (DERAA) em pacientes com AR e grupo-controle

\begin{tabular}{llll}
\hline Grupo & Genótipo HLA-DRB1 & AR $=\mathbf{4 1 2}, \mathbf{n}(\mathbf{\%})$ & Controle $=\mathbf{2 1 5}, \mathbf{n}(\%)$ \\
\hline A & EC/EC & $45(10,9)$ & $11(5,1)$ \\
B & EC/X & $175(43,2)$ & $42(19,5)$ \\
C & EC/DERAA & $35(7,7)$ & $14(6,5)$ \\
D & X/X & $110(26,6)$ & $84(39,1)$ \\
E & X/DERAA & $43(10,4)$ & $57(26,5)$ \\
F & DERAA/DERAA & $4(0,9)$ & $7(3,3)$ \\
\hline
\end{tabular}

Alelos EC são HLA-DRB1 ${ }^{*} 01: 01,{ }^{*} 01: 02,{ }^{*} 04: 01,{ }^{*} 04: 04,{ }^{*} 04: 05,{ }^{*} 04: 08,{ }^{*} 10: 01 \mathrm{e}$ *14:02. Alelos DERAA são HLA-DRB1 ${ }^{*} 01: 03,{ }^{*} 04: 02,{ }^{*} 11: 02,{ }^{*} 11: 03,{ }^{*} 13: 01,{ }^{*} 13: 02 \mathrm{e}$ *13:04. Alelos X são todos os outros alelos HLA-DRB1.

OR, IC 95\% e valor P dos seguintes dados: Grupo B comparado ao grupo C: OR 0,60; IC 95\% 0,28-1,29; P= 0,216. Grupo D comparado aos grupos E e F: OR 0,56; IC 95\% 0,34-0,92; P = 0,021. Grupo A mais B comparado ao grupo D: OR 3,17; IC 95\% 2,05-4,90; $P=0,00$.

\section{Tabela 6}

Frequência de alelos HLA-DRB1 EC e autoanticorpos em 412 pacientes com AR

\begin{tabular}{|c|c|c|c|c|c|}
\hline Autoanticorpos & $E C+, n(\%)$ & EC-, n(\%) & OR & $95 \%$ IC & $\mathbf{P}$ \\
\hline Fator reumatoide & & & 1,26 & $0,82-1,94$ & 0,313 \\
\hline Positivo & $165(40,04)$ & $92(22,33)$ & & & \\
\hline Negativo & $91(22,08)$ & $64(15,53)$ & & & \\
\hline ACPA & & & 2,03 & $1,28-3,31$ & 0,001 \\
\hline Positivo & $197(47,81)$ & $97(23,54)$ & & & \\
\hline Negativo & $59(14,32)$ & $59(14,32)$ & & & \\
\hline
\end{tabular}

EC+: epítopo compartilhado positivo; EC-: epítopo compartilhado negativo; ACPA: anticorpos antipeptídeo citrulinado; OR: razão de risco.

\section{Associação entre alelos HLA-DRB1 de} suscetibilidade (EC) e presença de autoanticorpos

Os pacientes com AR apresentaram FR positivo em 62,3\% $(n=257)$ e ACPA positivo em 71,3\% $(n=294)$ dos casos, dos quais $237(57,5 \%)$ dos pacientes foram positivos para ambos os autoanticorpos e somente 57 pacientes $(13,8 \%)$ foram positivos apenas para o ACPA. Observamos também uma associação significativa entre FR e ACPA (OR = 20,79; IC 95\% 11,49-37,97; P < 0,001).

Expressavam FR+ e alelos HLA-DRB1 EC+ 165 (40,04\%) pacientes, e 197 (47,81\%) expressavam ACPA+ e alelos HLA-DRB1 EC+. Associação significativa foi observada somente para alelos EC com ACPA (Tabela 6).

Os níveis séricos do FR para o grupo de pacientes com genótipos EC homozigotos, heterozigotos e sem o EC foram $177,7 \pm 286 \mathrm{UI} / \mathrm{mL}, 205,3 \pm 695 \mathrm{UI} / \mathrm{mL}$ e $157,1 \pm 328 \mathrm{UI} / \mathrm{mL}$, respectivamente. Os níveis séricos médios para o ACPA, seguindo a mesma ordem do FR, foram 131,3 $\pm 66 \mathrm{U} / \mathrm{mL}, 102,8 \pm 71 \mathrm{U} / \mathrm{mL}$ e $83,3 \pm 73 \mathrm{U} / \mathrm{mL}$, respectivamente.

\section{DISCUSSÃO}

Estudos genéticos realizados inicialmente em gêmeos e posteriormente em familiares demonstraram uma predisposição familiar para AR, representando $60 \%$ do risco total para o desenvolvimento da doença na população. ${ }^{4}$ Diversos estudos demonstraram que o fator genético mais importante associado à suscetibilidade da AR é a presença do HLA-DRB1 do MHC, descoberto inicialmente por Stastny et al. ${ }^{34}$ e posteriormente organizado na hipótese do EC por Gregersen et al. ${ }^{6}$

Técnicas modernas como as empregadas em estudos de genoma completo (GWAS, do inglês, genome-wide association studies) e marcadores genéticos de polimorfismo de único nucleotídeo (SNP, do inglês, single nucleotide polymorphism) confirmam a associação significante e preponderante desses alelos com a AR. No entanto, é importante ressaltar que a associação da AR ao HLA-DRB1 e a hipótese do EC não explicam toda a suscetibilidade genética conferida pelo HLA. Outros genes do HLA, sem EC, tais como os alelos HLA-DRB1*03:01, DRB1*07:01 e DRB1*09:01, embora com frequência menor, também foram associados à maior suscetibilidade para a AR..$^{35,36}$ 
Um segundo gene associado à doença é a proteína tirosina fosfatase N22 (PTPN22, do inglês, protein tyrosine phosphatase), presente em $8 \%$ dos pacientes com AR. ${ }^{32}$ Em menor proporção, foram descritas associações a determinados alelos do sinal transdutor e ativador de transcrição 4 (STAT4, do inglês, signal transducer and activator of transcription 4), do antígeno 4 dos linfócitos T citotóxicos (CTLA-4, do inglês, cytotoxic T-lymphocyte antigen), do fator inibitório de migração de macrófagos (MIF, do inglês, macrophag emigration inhibitory factor) e da peptidilarginina deaminase 4 (PADI4, do inglês, peptidylarginine deiminase type IV). ${ }^{37}$

No presente estudo observamos que $90,8 \%$ dos pacientes eram mulheres, semelhante à proporção encontrada no estudo realizado por Bertolo et al. ${ }^{22} \mathrm{em} 2001$ (84,6\%) e por LouzadaJunior et al. ${ }^{23}$ em 2008 (77,8\%).

A influência da etnia é ponto fundamental quando se avalia a associação dos genes HLA com doenças, especialmente com AR. Além de ser um sistema altamente polimórfico, distintos alelos são associados à AR em diferentes populações. Dessa forma, ao estudar uma população altamente miscigenada como a brasileira, as associações tradicionais de determinadas etnias poderiam não ser verificadas. No intuito de controlar essa variável, todo estudo de associação HLA e doença necessita que os indivíduos-controle sejam oriundos do mesmo estrato sociogeográfico dos pacientes, a fim de preservar as influências sociais e demográficas em ambos os grupos. Nosso estudo, além de avaliar uma amostra significativa de pacientes com AR $(\mathrm{n}=412)$, também tipificou indivíduos saudáveis $(\mathrm{n}=215)$, todos provenientes da mesma região geográfica e estrato social. Além disso, houve predomínio de mestiços, seguido de brancos e negros, configurando-se uma amostra altamente miscigenada (descendentes principalmente de portugueses, africanos, indígenas, italianos, espanhóis e alemães), que acreditamos refletir, em parte, a própria população brasileira.

Em princípio, a idade em estudos genéticos não teria influência na suscetibilidade. Porém, em virtude da presença de alelos HLA-DRB1 com EC+ entre $12,5 \%$ e $35 \%^{23,38}$ da população sadia e da incidência maior da AR entre 30 e 55 anos, ${ }^{2}$ tentamos evitar viés de análise quanto ao diagnóstico no grupo-controle. Por isso, decidimos incluir em nosso grupo-controle indivíduos com idade mais avançada.

Com relação à associação do HLA-DRB1 com a doença, observamos frequência aumentada dos alelos HLA-DRB1*04:01, *04:04 e *04:05 e associação positiva com a suscetibilidade à AR. Este resultado está, em parte, de acordo com os observados por Louzada-Junior et al. ${ }^{23}$ na população brasileira predominantemente caucasiana, na qual, além da associação aos alelos citados anteriormente, também foi encontrada associação ao alelo HLA-DRB1*01:01. Por outro lado, difere da observada por Bertolo et al., ${ }^{22}$ que também analisaram populações de origem caucasiana e observaram associação significativa somente com alelos HLA-DRB1*01 (OR = 2,8).

Assim como observado por Louzada-Junior et al., ${ }^{23} \mathrm{o}$ alelo HLA-DRB1*14:02 mostrou tendência de associação à $\mathrm{AR}$, embora de forma não significativa $(\mathrm{OR}=3,18$; IC $95 \%$ 0,88-13,63; $\mathrm{P}=0,086)$. É possível que estudos com maior número de indivíduos possam mostrar que essa associação seja estatisticamente significante. Em um estudo com população mestiça peruana $(n=65)$ essa associação entre o alelo HLA-DRB1*14:02 e a AR foi observada $(\mathrm{OR}=2,74) .{ }^{18}$

A presença dos alelos HLA-DRB $1 * 09$ em $6,7 \%$ dos pacientes com AR e 1,3\% dos controles, predominante em descendentes de negros e indígenas, mostrou associação significativa nos genótipos heterozigotos desses alelos $(\mathrm{OR}=5,19$; IC 95\% 1,50$21,49 ; \mathrm{P}=0,004)$. Não obstante o amplo intervalo de confiança observado, este alelo foi pela primeira vez associado à suscetibilidade à AR na população brasileira. Essa mesma associação (HLA-DRB1*09 e AR) também foi observada em pacientes chilenos com AR, em 1990. ${ }^{39}$ Por outro lado, estudo realizado no Japão com 852 pacientes com AR encontrou associação com a doença apenas no genótipo HLA-DRB $1 * 09: 01$ homozigoto. ${ }^{36}$

Semelhante aos estudos de Vignal et al..$^{35}(\mathrm{OR}=5,04) \mathrm{e}$ Balsa et al..$^{38}(\mathrm{OR}=1,8)$ incluindo população etnicamente homogênea, também encontramos associação do conjunto de alelos HLA-DRB1 $\mathrm{EC}+$ com a AR $(\mathrm{OR}=3,59)$. Este resultado é distinto ao descrito no estudo realizado por Teller et al. ${ }^{40}$ incluindo pacientes com AR e controles hispano-americanos, no qual não foi observada associação desses alelos com a doença, o que sugere que a hipótese do EC provavelmente não poderia ser aplicada em estudos de população não miscigenada.

No presente estudo pode-se observar frequência aumentada de genótipos EC heterozigotos (51\%) quando comparados aos pacientes com AR e controle com genótipos EC homozigotos (11\%). Em estudo realizado por del Rincon et al. ${ }^{41}$ foram observados genótipos EC heterozigotos em 52\% dos pacientes com AR, e EC homozigotos em $22 \%$. Entretanto, Balsa et al. ${ }^{38}$ encontraram que $29,8 \%$ dos pacientes eram heterozigotos e $14 \%$ eram homozigotos. Neste estudo, após análise de regressão logística dos genótipos homozigotos e heterozigotos HLA-DRB1 com EC+, observamos que pacientes que apresentam $\mathrm{EC}$, independente de serem homozigotos $(\mathrm{OR}=3,86)$ ou heterozigotos $(\mathrm{OR}=3,54)$, apresentam risco aumentado, mas de magnitude semelhante (OR semelhantes) para o desenvolvimento da AR. Este resultado se contrapõe ao de del Rincon et al., ${ }^{41}$ no qual a análise de 141 pacientes mexicanos com AR mostrou maior risco para genótipos EC 
homozigotos $(\mathrm{OR}=21,53)$ quando comparados com genótipos EC heterozigotos $(\mathrm{OR}=1,84)$.

Com relação aos alelos HLA-DRB1 protetores para AR, observamos que alelos HLA-DRB1 codificadores da sequência de aminoácidos DERAA estão associados a menor risco de desenvolver AR $(\mathrm{OR}=0,42)$, semelhante ao observado por Louzada-Junior et al. ${ }^{23} \mathrm{em} 2008(\mathrm{OR}=0,49)$. Carrier et al. ${ }^{25}$ avaliaram pacientes com poliartrite de início recente e obsevaram que os alelos DERAA não estavam associados à produção de autoanticorpos, bem como também mostrou-se efeito protetor para o desenvolvimento da $\mathrm{AR}(\mathrm{OR}=0,30)$. Mais recentemente, Balsa et al ${ }^{38}$ relataram que esses alelos conferem proteção para $\mathrm{AR}$ apenas com ACPA circulante $(\mathrm{OR}=0,58)$. Outro estudo mostrou que alelos DERAA, além de conferir efeito protetor para $\mathrm{AR}$, estariam associados à enfermidade menos grave. ${ }^{24}$

Observamos maior frequência de FR (62\%) e ACPA (71\%) positivos quando comparados com grupo de pacientes brasileiros com AR inicial, ${ }^{42}$ cujas frequências eram em torno de $50 \%$. Tal resultado pode ser justificado devido ao nosso grupo de pacientes apresentar maior tempo de doença (em torno de nove anos). Alelos HLA-DRB1 com EC foram relacionados com a positividade do FR e do ACPA, embora só a associação com a presença do ACPA tenha se mostrado significante (Tabela 6). Irigoyen et al., ${ }^{29} \mathrm{em}$ 2005, observaram forte associação do ACPA a alelos EC, independentemente da presença do FR (OR = 5,8; IC 95\% $4,1-8,3 ; \mathrm{P}<0,001$ e OR = 3,1; IC 95\% 1,8-5,3; $\mathrm{P}<0,001)$. Semelhante ao observado por Balsa et al., ${ }^{38}$ este estudo mostrou que genótipos HLA-DRB1 EC homozigotos apresentaram níveis séricos do ACPA mais elevados quando comparados aos genótipos heterozigotos e aos dos pacientes sem o EC. Em contraste, os níveis séricos de FR não diferiram em pacientes com e sem alelos EC. Observamos que $88,8 \%$ de 27 pacientes com alelo HLA-DRB $1 * 09$ apresentaram ACPA e $85 \%$ apresentaram FR, sugerindo que outros mecanismos não EC estariam implicados no risco genético para o desenvolvimento de FR e ACPA.

Em síntese, este estudo, com amostra populacional predominantemente mestiça e mais representativa do povo brasileiro, evidenciou que os alelos HLA-DRB1*04:01, *04:04 e *04:05 associaram-se à suscetibilidade aumentada para AR, destacando-se também a associação ao alelo DRB1*09 nesses pacientes. Nossos resultados corroboram a associação entre alelos DRB1 EC e a suscetibilidade à AR e ao ACPA, previamente documentada em estudos com amostras populacionais geneticamente homogêneas. Além disso, mostramos que a presença do EC, quer em dose única ou dupla, comportou-se como fator de risco independente para a doença, bem como que a presença de alelos DERAA apresentaram efeito protetor. Ademais, os alelos HLA-EC estiveram associados a maior positividade e maiores níveis séricos de ACPA. Embora as diretrizes para o diagnóstico da AR estabelecidas pela Sociedade Brasileira de Reumatologia em $2011^{43}$ recomendem que a pesquisa do HLA-EC não deva ser ainda um exame de rotina diária ao atendimento de pacientes com suspeita de AR, devido ao seu custo elevado, nosso estudo corrobora sua importância para o estabelecimento de fatores de risco e de proteção ao desenvolvimento da $\mathrm{AR}$.

\section{REFERENCES}

\section{REFERÊNCIAS}

1. Senna ER, de Barros AL, Silva EO, Costa IF, Pereira LV, Ciconelli RM et al. Prevalence of rheumatic diseases in Brazil: a study using the COPCORD approach. J Rheumatol 2004; 31(3):594-7.

2. Pinheiro GRC. Artrite reumatoide. In: Moreira C, Pinheiro GRC, Marques Neto JF (eds.). Reumatologia Essencial. Rio de Janeiro: Guanabara Koogan; 2009. p. 338-54.

3. Hochberg MC, Silman AJ, Smolen JS, Weinblatt ME, Weisman MH. Rheumatology. 4ed. Philadelphia: Mosby; 2008.

4. MacGregor AJ, Snieder H, Rigby AS, Koskenvuo M, Kaprio J, Aho $\mathrm{K}$ et al. Characterizing the quantitative genetic contribution to rheumatoid arthritis using data from twins. Arthritis Rheum 2000; 43(1):30-7.

5. Bowes J, Barton A. Recent advances in the genetics of RA susceptibility. Rheumatology (Oxford) 2008; 47(4):399-402.

6. Gregersen PK, Silver J, Winchester RJ. The shared epitope hypothesis. An approach to understanding the molecular genetics of susceptibility to rheumatoid arthritis. Arthritis Rheum 1987; 30(11):1205-13.

7. Klareskog L, Stolt P, Lundberg K, Kallberg H, Bengtsson C, Grunewald $\mathrm{J}$ et al. A new model for an etiology of rheumatoid arthritis: smoking may trigger HLA-DR (shared epitope)-restricted immune reactions to autoantigens modified by citrullination. Arthritis Rheum 2006; 54(1):38-46.

8. Weyand CM XC, Goronzy JJ. Homozygosity for the HLA-DRB1 allele selects for extra-articular manifestations in rheumatoid arthritis. J Clin Invest1992; 89(6):2033-9.

9. Weyand CM, Goronzy JJ. Inherited and noninherited risk factors in rheumatoid arthritis. Curr Opin Rheumatol 1995; 7(3):206-13.

10. Massardo L, Gareca N, Cartes MA, Cervilla V, GonzalezA, Jacobelli S. The presence of the HLA-DRB1 shared epitope correlates with erosive disease in Chilean patients with rheumatoid arthritis. Rheumatology (Oxford) 2002; 41(2):153-6.

11. Morgan AW, Haroon-Rashid L, Martin SG, Gooi HC, Worthington J, Thomson W et al. The shared epitope hypothesis in rheumatoid arthritis: evaluation of alternative classification criteria in a large UK Caucasian cohort. Arthritis Rheum 2008; 58(5):1275-83.

12. Gonzalez-Gay MA, Garcia-Porrua C, Hajeer AH. Influence of human leukocyte antigen-DRB1 on the susceptibility and severity of rheumatoid arthritis. Semin Arthritis Rheum 2002; 31(6):355-60.

13. Lee HS, Lee KW, Song GG, Kim HA, Kim SY, Bae SC. Increased susceptibility to rheumatoid arthritis in Koreans heterozygous for HLA-DRB1*0405 and *0901. Arthritis Rheum 2004; 50(11):3468-75. 
14. Wakitani S, Murata N, Toda Y, Ogawa R, Kaneshige T, Nishimura Y et al. The relationship between HLA-DRB1 alleles and disease subsets of rheumatoid arthritis in Japanese. Br J Rheumatol 1997; 36(6):630-6.

15. de Vries N, Ronningen KS, Tilanus MG, Bouwens-Rombouts A, Segal R, Egeland T et al. HLA-DR1 and rheumatoid arthritis in Israeli Jews: sequencing reveals that DRB $1 * 0102$ is the predominant HLA-DR1 subtype. Tissue Antigens 1993; 41(1):26-30.

16. Boki KA, Panayi GS, Vaughan RW, Drosos AA, Moutsopoulos HM, Lanchbury JS. HLA class II sequence polymorphisms and susceptibility to rheumatoid arthritis in Greeks. The HLA-DR beta shared-epitope hypothesis accounts for the disease in only a minority of Greek patients. Arthritis Rheum 1992; 35(7):749-55.

17. Templin DW, Boyer GS, Lanier AP, Nelson JL, Barrington RA, Hansen JA et al. Rheumatoid arthritis in Tlingit Indians: clinical characterization and HLA associations. J Rheumatol 1994; 21(7):1238-44.

18. Castro F, Acevedo E, Ciusani E, Angulo JA, Wollheim FA, Sandberg-Wollheim M. Tumour necrosis factor microsatellites and HLA-DRB1*, HLA-DQA1*, and HLA-DQB1* alleles in Peruvian patients with rheumatoid arthritis. Ann Rheum Dis 2001; 60(8):791-5.

19. Arias MV, Domingues EV, Lozano RB, Flores CV, Peralta MM, Salinas CZ. Study of class I and II HLA alleles in 30 Ecuadorian patients with rheumatoid arthritis compared with alleles from healthy and affected subjects with other rheumatic diseases. Rev Bras Reumatol 2010; 50(4):423-33.

20. Anaya JM, Correa PA, Mantilla RD, Arcos-Burgos M. Rheumatoid arthritis association in Colombian population is restricted to HLA-DRB1*04 QRRAA alleles. Genes Immun 2002; 3(1):56-8.

21. Citera G, Padulo LA, Fernandez G, Lazaro MA, Rosemffet MG, Maldonado Cocco JA. Influence of HLA-DR alleles on rheumatoid arthritis: susceptibility and severity in Argentine patients. J Rheumatol 2001; 28(7):1486-91.

22. Bertolo MB, Costallat LTL, Persoli LB, Costa FF. Alelos HLA-DRB1 e prognóstico da artrite reumatoide em pacientes brasileiros. Rev Bras Reumatol 2001; 41(3):151-6.

23. Louzada-Junior P, Freitas MVC, Oliveira RDR, Deghaide NHS, Conde RA, Bertolo MB et al. A majority of Brazilian patients with rheumatoid arthritis HLA-DRB1 alleles carry both the HLA-DRB1 shared epitope and anti-citrullinated peptide antibodies. Braz J Med Biol Res 2008; 41(6):493-9.

24. van der Helm-van Mil AH, Huizinga TW, Schreuder GM, Breedveld FC, de Vries RR, Toes RE. An independent role of protective HLA class II alleles in rheumatoid arthritis severity and susceptibility. Arthritis Rheum 2005; 52(9):2637-44.

25. Carrier N, Cossette P, Daniel C, de Brum-Fernandes A, Liang P, Menard HA et al. The DERAA HLA-DR alleles in patients with early polyarthritis: protection against severe disease and lack of association with rheumatoid arthritis autoantibodies. Arthritis Rheum 2009; 60(3):698-707.

26. Bukhari M, Lunt M, Harrison BJ, Scott DG, Symmons DP, Silman AJ. Erosions in inflammatory polyarthritis are symmetrical regardless of rheumatoid factor status: results from a primary carebased inception cohort of patients. Rheumatology (Oxford) 2002; 41(3):246-52.

27. Meyer O, Labarre C, Dougados M, Goupille P, Cantagrel A, Dubois A et al. Anticitrullinated protein/peptide antibody assays in early rheumatoid arthritis for predicting five year radiographic damage. Ann Rheum Dis 2003; 62(2):120-6.
28. Mattey DL, Dawes PT, Clarke S, Fisher J, Brownfield A, Thomson W et al. Relationship among the HLA-DRB1 shared epitope, smoking, and rheumatoid factor production in rheumatoid arthritis. Arthritis Rheum 2002; 47(4):403-7.

29. Irigoyen P, Lee AT, Wener MH, Li W, Kern M, Batliwalla F et al. Regulation of anti-cyclic citrullinated peptide antibodies in rheumatoid arthritis: contrasting effects of HLA-DR3 and the shared epitope alleles. Arthritis Rheum 2005; 52(12):3813-8.

30. Orozco G, Pascual-Salcedo D, Lopez-Nevot MA, Cobo T, Cabezon A, Martin-Mola E et al. Auto-antibodies, HLA and PTPN22: susceptibility markers for rheumatoid arthritis. Rheumatology (Oxford) 2008; 47(2):138-41.

31. van der Helm-van Mil AH, Verpoort KN, Breedveld FC, Huizinga TW, Toes RE, de Vries RR. The HLA-DRB1 shared epitope alleles are primarily a risk factor for anti-cyclic citrullinated peptide antibodies and are not an independent risk factor for development of rheumatoid arthritis. Arthritis Rheum 2006; 54(4):1117-21.

32. Klareskog L, Catrina AI, Paget S. Rheumatoid arthritis. Lancet 2009; 373(9664):659-72.

33. Arnett FC, Edworthy SM, Bloch DA, McShane DJ, Fries JF, Cooper NS et al. The American Rheumatism Association 1987 revised criteria for the classification of rheumatoid arthritis. Arthritis Rheum 1988; 31(3):315-24.

34. Stastny P. Association of the B-cell alloantigen DRw4 with rheumatoid arthritis. N Engl J Med 1978; 298(16):869-71.

35. Vignal C, Bansal AT, Balding DJ, Binks MH, Dickson MC, Montgomery DS et al. Genetic association of the major histocompatibility complex with rheumatoid arthritis implicates two non-DRB1 loci. Arthritis Rheum 2009; 60(1):53-62.

36. Wakitani S, Imoto K, Murata N, Toda Y, Ogawa R, Ochi T. The homozygote of HLA-DRB1*0901, not its heterozygote, is associated with rheumatoid arthritis in Japanese. Scand J Rheumatol 1998; 27(5):381-2.

37. van der Helm-van Mil AH, Huizinga TW. Advances in the genetics of rheumatoid arthritis point to subclassification into distinct disease subsets. Arthritis Res Ther 2008; 10(2):205.

38. Balsa A, Cabezon A, Orozco G, Cobo T, Miranda-Carus E, Lopez-Nevot MA et al. Influence of HLA-DRB1 alleles in the susceptibility of rheumatoid arthritis and the regulation of antibodies against citrullinated proteins and rheumatoid factor. Arthritis Res Ther 2010; 12(2):R62.

39. Gonzalez A, Nicovani S, Massardo L, Aguirre V, Cervilla V, Lanchbury JS et al. Influence of the HLA-DR beta shared epitope on susceptibility to and clinical expression of rheumatoid arthritis in Chilean patients. Ann Rheum Dis 1997; 56(3):191-3.

40. Teller K, Budhai L, Zhang M, Haramati N, Keiser HD, Davidson A. HLA-DRB1 and DQB typing of Hispanic American patients with rheumatoid arthritis: the "shared epitope" hypothesis may not apply. J Rheumatol 1996; 23(8):1363-8.

41. del Rincon I, Escalante A. HLA-DRB1 alleles associated with susceptibility or resistance to rheumatoid arthritis, articular deformities, and disability in Mexican Americans. Arthritis Rheum 1999; 42(7):1329-38.

42. da Mota LM, dos Santos Neto LL, Burlingame R, Menard HA, Laurindo IM. Laboratory characteristics of a cohort of patients with early rheumatoid arthritis. Rev Bras Reumatol 2010; 50(4):375-88.

43. da Mota LM, Cruz BA, Brenol CV, Pereira IA, Fronza LS, Bertolo MB et al. 2011 Consensus of the Brazilian Society of Rheumatology for diagnosis and early assessment of rheumatoid arthritis. Rev Bras Reumatol 2011; 51(3):199-219. 